\title{
Special volume dedicated to Ronald Brown for his 80th birthday
}

\author{
Maria Manuel Clementino' ${ }^{1}$ Marino Gran² . \\ George Janelidze $^{3}$. Timothy Porter ${ }^{4}$
}

Published online: 27 October 2016

(C) Tbilisi Centre for Mathematical Sciences 2016

This volume of Journal of Homotopy and Related Structures is dedicated to Professor Ronald Brown on the occasion of his 80th birthday, in appreciation of his so distinguished and beautiful contribution to algebraic topology, homotopical algebra, and related areas of mathematics.

Ronnie's research begins with several diverse topics of topology and homological algebra, including the study of product topologies and what would later be described as monoidal closed structures on the category of topological spaces, then Künneth relations, the homotopy theory of fibrations, and the sequential approach to topology, to mention only a few. His later work included developing computational methods for perturbing a homotopy type, and already in 1967, he showed the advantages in using

Communicated by Maria Manuel Clementino, Marino Gran, George Janelidze and Timothy Porter.

George Janelidze

george.janelidze@uct.ac.za

Maria Manuel Clementino

mmc@mat.uc.pt

Marino Gran

marino.gran@uclouvain.be

Timothy Porter

t.porter.maths@gmail.com

1 CMUC, Department of Mathematics, University of Coimbra, 3001-501 Coimbra, Portugal

2 Institut de Recherche en Mathématique et Physique, Université catholique de Louvain, Chemin du Cyclotron 2, 1348 Louvain-la-Neuve, Belgium

3 Department of Mathematics and Applied Mathematics, University of Cape Town, Rondebosch 7701, South Africa

4 Bangor, North Wales, UK 
groupoids in proofs of variants of van Kampen's theorem. His first book, published in 1967, introduced both categorical arguments and groupoid theory as algebra to many topologists. In 1970, he moved from Hull to Bangor and later in the 1970s started his very fruitful collaboration with Philip J. Higgins. In this collaboration, and in collaboration with many others, as well as just by himself, we find work on topological groupoids, crossed modules, then $\omega$-groupoids and higher-dimensional algebra, together with applications of these to combinatorial and cohomological group theory which have all been studied in the process, pushing forward into unknown mathematical territory.

We would also like to mention his well-documented exchanges that resulted in Grothendieck's Pursuing Stacks that typifies the innovative way that Ronnie reacts to new situations. He has had a very wide influence in all of the areas in which he has worked, and has been generous with his help and advice to the mathematical community.

We thank Hvedri Inassaridze and all the editors of JHRS for giving us the opportunity to publish this volume, and we join with the authors to offer Ronnie these articles to celebrate his 80th birthday, even if his 81st has now passed!

Maria Manuel Clementino, Marino Gran, George Janelidze, Timothy Porter

July 2016

\section{List of Publications of Ronald Brown}

DPhil Thesis: 'Some problems in algebraic topology: a study of function space, function complexes, and FD-complexes', 1961, 140 pages, (Supervised by M. G. Barratt).

\section{Books}

a. "Elements of Modern Topology", McGraw Hill, Maidenhead, 1968 (out of print in 1972).

b. (coedited with T. L. Thickstun), "Low-Dimensional Topology", (Volume 1 of the conference on Topology in Low Dimension, Bangor 1979), London Math. Soc. Lecture Notes No. 48 (1982).

c. "Topology: a geometric account of general topology, homotopy types, and the fundamental groupoid", Ellis Horwood, Chichester (1988) 460 pp. revised edition of (a). (out of print in 1992).

d. "Topology and Groupoids", Booksurge PLC, 2006, (Amazon), revised and updated edition of (c).

e. "Nonabelian algebraic topology, filtered space, crossed complexes, cubical homotopy groupoids", with P. J. Higgins and R. Sivera, EMS Tracts in Mathematics, vol. 15, (2011).

\section{Papers (Mathematics and related areas)}

1. “Ten topologies for $X \times Y$ ", Quart. J. Math. (2) 14 (1963), 303-319.

2. "Function spaces and product topologies", Quart. J. Math. (2) 15 (1964), 238250 . 
3. "Cohomology with chains as coefficients", Proc. London Math. Soc. (3) 14 (1964), 545-565.

4. "On Künneth suspensions", Proc. Camb. Phil. Soc. 60 (1964) 713-720.

5. "The twisted Eilenberg-Zilber theorem", Celebrazioni Archimedi de secolo xx, Syracusa, 1964, Simposi di topologia (1967) 33-37.

6. "On a method of P. Olum", J. London Math. Soc. 40 (1965), 303-304.

7. "Two examples in homotopy theory", Proc. Camb. Phil. Soc. 621 (1966) 575576.

8. “Groupoids and Van Kampen's theorem”, Proc. London Math. Soc. (3) 17 (1967) 385-401.

9. (with P. R. Heath), "Coglueing homotopy equivalences”, Math. Z. 113 (1970) 313-362.

10. "Fibrations of groupoids", J. Algebra 15 (1970) 103-132.

11. "The calculus and modern mathematics", Mathematics Teaching 51 (1970) 6 pp.

12. "Groupoids as coefficients", Proc. London Math Soc. (3) 25 (1972) 413-426.

13. "Sequentially proper maps and a sequential compactification", J. London Math Soc. (2) 7 (1973) 515-522.

14. (with G. Danesh-Naruie), "The fundamental groupoid as a topological groupoid", Proc. Edinburgh Math. Soc. 19 (1975) 237-244.

15. (with J. P. L. Hardy), "Subgroups of free topological groups and free topological products of topological groups", J. London Math Soc. (2) 10 (1975) 431-440.

16. (with P. J. Higgins and S. A. Morris), "Countable products of lines and circles: their closed subgroups, quotients and duality properties", Math. Proc. Camb. Phil. Soc. 78 (1975) 19-32.

17. "Some non-projective subgroups of free topological groups", Proc. Amer. Math. Soc. 52 (1975) 443-440.

18. (with J. P. L. Hardy and S. A. Morris), "The homotopy groups of an $n$-fold wedge", Math. Z. 143 (1975) 119-121.

19. (with J. P. L. Hardy), "Topological groupoids I: universal constructions", Math. Nachr. 71 (1976) 273-286.

20. (with G. Danesh-Naruie and J. P. L. Hardy), “Topological groupoids II: covering morphisms and -spaces", Math. Nachr. 74 (1976) 143-156.

21. (with C. B. Spencer), " $\mathcal{G}$-groupoids, crossed modules and the fundamental groupoid of a topological group", Proc. Kon. Ned. Akad. v. Wet. 7 (1976) 296302.

22. (with C. B. Spencer), "Double groupoids and crossed modules", Cah. Top. Géom. Diff. 17 (1976) 343-362.

23. (with P. J. Higgins), "Sur les complexes croisés, $\omega$-groupodes et $T$-complexes", C.R. Acad. Sci. Paris Sér. A. 285 (1977) 997-999.

24. (with P. J. Higgins), "Sur les complexes croisés d'homotopie associés a quelques espaces filtrés”, C. R. Acad. Sci. Paris Sér. A.286 (1978) 91-93.

25. (with S. A. Morris), "Embeddings in contractible or compact objects", Coll. Math. 38 (1978) 193-212.

26. (with P. J. Higgins), "On the connection between the second relative homotopy groups of some related spaces”, Proc. London Math. Soc. (3) 36 (1978) 193-212. 
27. (with P. I. Booth), "Spaces of partial maps, fibred mapping spaces and the compact-open topology", Gen. Top. Appl. 8 (1978) 181-195.

28. (with P. I. Booth), "On the application of fibred mapping spaces to exponential laws for bundles, ex-spaces and other categories of maps", Gen. Top. Appl. 8 (1978) 165-179.

29. (with P. Nickolas), "Exponential laws for topological categories, groupoids and groups and mapping spaces of colimits", Cah. Top. Géom. Diff. 20 (1979) 179_ 198.

30. (with A. M. Abd-Allah), "A compact-open topology on partial maps with open domain", J. London Math Soc. (2) 21 (1980) 480-486.

31. "On the second relative homotopy group of an adjunction space: an exposition of a theorem of J.H.C. Whitehead", J. London Math. Soc. (2) 22 (1980) 146-152.

32. (with P. J. Higgins), “On the algebra of cubes”, J. Pure Appl. Algebra 21 (1981) 233-260.

33. (with P. J. Higgins), "Colimit theorems for relative homotopy groups", J. Pure Appl. Algebra 22 (1981) 11-41.

34. (with P. J. Higgins), "The equivalence of $\Omega$-groupoids and cubical $T$-complexes", Cah. Top. Géom. Diff. 22 (1981) 349-370.

35. (with P. J. Higgins), "The equivalence of $\infty$-groupoids and crossed complexes", Cah. Top. Géom. Diff. 22 (1981) 371-386.

36. (with J. Huebschmann), "Identities among relations", in Low dimensional topology, London Math. Soc. Lecture Note Series 48 (ed. R. Brown and T.L. Thickstun, Cambridge University Press, 1982), pp. 153-202.

37. "Higher dimensional group theory", in Low dimensional topology, London Math Soc. Lecture Note Series 48 (ed. R. Brown and T.L. Thickstun, Cambridge University Press, 1982), pp. 215-238.

38. (with P. J. Higgins), "Crossed complexes and non-abelian extensions", Category theory proceedings, Gummersbach, 1981, Lecture Notes in Math. 962 (ed. K.H. Kamps et al, Springer, Berlin, 1982), pp. 39-50.

39. "Non-abelian cohomology and the homotopy classification of maps", in Homotopie algébrique et algebre locale, Conf. Marseille-Luminy 1982, ed. J.-M. Lemaire et J.-C. Thomas, Astérisques 113-114 (1984), 167-172.

40. "An introduction to simplicial T-complexes", in Esquisses Math., 32 (1982) 127.

41. (with P. R. Heath and K. H. Kamps), "Groupoids and the Mayer-Vietoris sequence", J. Pure Appl. Alg. 30 (1983) 109-129.

42. (with A. Razak Salleh), "A van Kampen theorem for unions of non-connected spaces", Archiv. Math. 42 (1984) 85-88.

43. (with J.-L. Loday), "Excision homotopique en basse dimension", C.R. Acad. Sci. Paris Sér. I 298 (1984) 353-356.

44. "Coproducts of crossed $P$-modules: applications to second homotopy groups and to the homology of groups", Topology 23 (1984) 337-345.

45. (with P. R. Heath and K. H. Kamps), "Coverings of groupoids and Mayer-Vietoris type sequences”, Categorical Topology, Proc. Conf. Toledo, Ohio, 1983. (Heldermann Verlag, Berlin) (1984) 147-162. 
46. "Some non-abelian methods in homotopy theory and homological algebra", Categorical topology: Proc. Conference Toledo, Ohio, 1983, (ed. H.L. Bentley et al., Heldermann, Berlin, 1984), pp. 108-146.

47. (with S. P. Humphries), "Orbits under symplectic transvections I", Proc. London Math. Soc. (3) 52 (1986) 517-531.

48. (with S. P. Humphries), "Orbits under symplectic transvections II: the case $K=$ $\mathbb{F}_{2}$ ”, Proc. London Math. Soc. (3) 52 (1986) 532-556.

49. (with P. J. Higgins), "Tensor products and homotopies for $\omega$-groupoids and crossed complexes", J. Pure Appl. Alg. 47 (1987) 1-33.

50. (with J.-L. Loday), "Homotopical excision, and Hurewicz theorems, for $n$-cubes of spaces", Proc. London Math. Soc. (3) 54 (1987) 176-192.

51. "From groups to groupoids: a brief survey", Bull. London Math. Soc. 19 (1987) 113-134.

52. (with J.-L. Loday), "Van Kampen theorems for diagrams of spaces", Topology 26 (1987) 311-334.

53. (with D. L. Johnson and E. F. Robertson), "Some computations of non-abelian tensor products of groups", J. Algebra 111 (1987) 177-202.

54. (with P. R. Heath), "Lifting amalgamated sums and other colimits of groups and topological groups", Math. Proc. Camb. Phil. Soc. 102 (1987) 273-280.

55. "A non-abelian tensor product of groups", Algebra-Tagung Halle 1986, Wissensch. Beitr., 33, Martin-Luther Univ. Halle-Wittenberg, Halle-Saale, (1987) $59-72$.

56. (with G. J. Ellis), "Hopf formulae for the higher homology of a group", Bull. London Math. Soc. 20 (1988) 124-128. plus erratum

57. "Carpentry: a fable", Mathematical Intelligencer, 11, no. 4 (1989) 37.

58. (with M. Golasiński), "A model structure for the homotopy theory of crossed complexes”, Cah. Top. Géom. Diff. Cat. 30 (1989) 61-82.

59. "Symmetry, groupoids, and higher dimensional analogues", Symmetry II, ed. Istvan Hargittai, Computers Math. Applic. 17 (1989) 49-57.

60. (with N. D. Gilbert), "Algebraic models of 3-types and automorphism structures for crossed modules", Proc. London Math. Soc. (3) 59 (1989) 51-73.

61. "Triadic Van Kampen theorems and Hurewicz theorems", Algebraic Topology, Proc. Int. Conf. March 1988, Edited M. Mahowald and S. Priddy, Cont. Math. 96 (1989) 39-57.

62. (with P. J. Higgins), "Crossed complexes and chain complexes with operators", Math. Proc. Camb. Phil. Soc. 107 (1990) 33-57.

63. "Some problems in non-Abelian homotopical and homological algebra", Homotopy theory and related topics, Proceedings Kinosaki, 1988, ed. M. Mimura, Springer Lecture Notes in Math., 1418 (1990) 105-129.

64. "Conversations with John Robinson" in Symbolism: sculptures and tapestries, by John Robinson, (Mathematics and Knots, Bangor, Wales) (1989) 2-29.

65. "Why we made an exhibition", in Souvenir Guide to the Pop Maths Road Show, Royal Society/Joint Mathematics Council (1989) 10-12.

66. (with T. Porter), "Making a mathematical exhibition", in The popularization of mathematics, edited A. G. Howson and J.-P. Kahane, ICMI Study Series, Cambridge University Press, (1990) 51-64. 
67. Review of Algebraic homotopy, by H. J. Baues, Cambridge University Press, 1988, 455 pp, Bull. American Math. Soc. 23 (1990) 182-190.

68. (with T. Porter), "Mathematics in Context: a new course", For the Learning of Mathematics, 10 (1990) 10-15.

69. " $q$-perfect groups and universal $q$-central extensions", Publ. Mat. Univ. Auton. Barcelona 34 (1990) 291-297.

70. "Local-to-global techniques, groupoids, and holonomy", Proceedings International Conference on the Calculus of Variations, Tbilisi Nov. 1989.

71. "Higher order symmetry", Proc. of Assiut First International Conference of Mathematics and Statistics, Assiut, Feb, 1990, Part I, Algebra and Geometry, Ed. E.K.Al-Hussaini and A.M.Abou-El-Ela, University of Assiut, (1990), 25-44.

72. (with P. J. Higgins), "The classifying space of a crossed complex", Math. Proc. Camb. Phil. Soc. 110 (1991) 95-120.

73. (with H. J. Baues), "On the relative homotopy groups of the product filtration and a formula of Hopf”, J. Pure Appl. Algebra 89 (1993) 49-61.

74. Preface to Combinatorial homotopy and four-dimensional complexes, by H. J. Baues, pp x-xx, de Gruyter, Berlin, 1991.

75. "Computing homotopy types using crossed $n$-cubes of groups", Adams Memorial Symposium on Algebraic Topology, Vol 1, edited N. Ray and G. Walker, Cambridge University Press, 1992, 187-210.

76. (with M. E.-S. A.-F. Aof), "The holonomy groupoid of a locally topological groupoid”, Top. Appl., 47 (1992) 97-113.

77. (with K. C. H. MacKenzie), "Determination of a double Lie groupoid by its core diagram”, J. Pure Appl. Algebra, 80 (1992) 237-272.

78. (with J. Robinson), "Borromean circles", Letter, American Math. Monthly, April, (1992) 376-377.

79. "Out of line", Royal Institution Proceedings, 64 (1992) 207-243.

80. (with J. Robinson), "The Universe Series of Symbolic Sculptures”, in Symbolic Sculptures, by John Robinson, Edition Limiteé, Carouge Geneve, (1992), 103123.

81. “The Waldegrave White Paper", London Math. Soc. Newsletter, November, (1992).

82. (with T. Porter), "Why choose mathematics?", in Mathematics for the Future, IMA and Hobson's Press, 1993, 1994, 1995).

83. (with O. Mucuk), "Covering groups of non-connected topological groups revisited”, Math. Proc. Camb. Phil. Soc, 115 (1994) 97-110.

84. (with C. Pritchard), "The third man's knotty problems", The Scottish Mathematical Council Journal, 23 (1993) 62-66.

85. "Symbolic sculptures by John Robinson", Bangor Tourist Guide, Mathematics and Knots, 1993.

86. "Higher order symmetry of graphs", Bull. Irish Math. Soc. 32 (1994) 46-59.

87. (with A. P. Tonks), "Calculations with simplicial and cubical groups in AXIOM", J. Symbolic Computation, 17 (1994) 159-179.

88. (with T. Porter), "The methodology of mathematics", Bulletin International Commission of Mathematical Instruction, 37 (1994) 23-37. 
89. (with O. Mucuk), "The monodromy groupoid of a Lie groupoid", Cah. Top. Géom. Diff. Cat,36 (1995) 345-369.

90. (with O. Mucuk), "Foliations, locally Lie groupoids, and holonomy", Cah. Top. Géom. Diff. Cat, 37 (1996) 61-71.

91. (with T. Porter), "The methodology of mathematics", Math. Gazette, 79, July (1995) 321-334. CUBO 2 (2000) 85-100.

92. "Representation and computation for crossed modules", Proceedings Catégories, Algebres, Esquisses, Neo-esquisses, Caen, 1994, 6 pp.

93. (with C. D. Wensley), "On finite induced crossed modules and the homotopy 2-type of mapping cones", Theory and Applications of Categories 1(3) (1995) $54-71$.

94. "Homotopy theory, and change of base for groupoids and multiple groupoids", Applied Categorical Structures, 4 (1996) 175-193.

95. (with M. Golasiński, T. Porter and A. P. Tonks), "Spaces of maps into classifying spaces for equivariant crossed complexes," Indag. Mathem., 8 (1997) 157-172.

96. (with C. D. Wensley), "Computing crossed modules induced by an inclusion of a normal subgroup, with applications to homotopy 2-types", Theory and Applications of Categories 2 (1996) 3-16.

97. (with G. Janelidze), "Van Kampen theorems for categories of covering morphisms in lextensive categories”, J. Pure Appl. Algebra 119 (1997) 255-263.

98. (with T. Porter), "On the Schreier theory of non-abelian extensions: generalisations and computations”, Proceedings Royal Irish Academy, 96 (1996) 213-227.

99. (with G. Janelidze), "Galois theory of second order covering maps of simplicial sets”, J. Pure Applied Algebra, 135 (1999) 83-91.

100. 'What should be the output of mathematical education?', in Mathematics Education as a research domain: a search for identity, edited Kilpatrick, J., Sierpinska, A., Kluwer, Lancaster, 1997, 459-476.

101. (with T. Porter), "Matematikos metodologia", Alfa + Omega, 1 Nr 5 (1998) 71-84 (Lithuanian translation of 'The Methodology of Mathematics'. Formally, "Alpha+omega" is a supplement of the "Lithuanian Mathematical Journal", ISSN number 0132-2818.)

102. 'Mathematics and knots', in Visual Representations and Interpretations, Ray Paton and Irene Nielson (Eds), Springer-Verlag London 1999, 32-42. [translated into Lithuanian as 'Matematika ir mazgai', “alpha + omega", No. 2 (6) (1998) $5-14$.

103. 'Groupoids and crossed objects in algebraic topology', Homology, homotopy and applications', 1 (1999) 1-78.

104. 'Nusos, matematiques i art', Butll. de la Soc. Catalana d. Matematiques, 13 (1998) 7-19. [Translated into Catalan by Gemma Bastardas from an article 'Knots, mathematics and art' published in English in the Actes del VI Congres de Llenguatges Naturels i Llenguatges Formals (Tarragona, 17-21 Sept, 1990).]

105. (with A. Razak Salleh), "Free crossed crossed resolutions of groups and presentations of modules of identities among relations", London Math. Soc. J. Computation and Math. (2) (1999) 28-61.

106. (with G. H. Mosa) 'Double categories, 2-categories, thin structures and connections', Theory and Applications of Categories, (1999), 163-175. 
107. 'John Robinson's Symbolic Sculptures, knots and mathematics', Proceedings ISAMA99, Ed N. Friedman and J. Barrallo, University of the Basque Country (San Sebastian) 1999, 75-82.

108. (with A. Heyworth) 'Using rewriting systems to compute left Kan extensions and induced actions of categories', J. Symbolic Computation 29 (2000) 5-31.

109. 'Dismay and decadence in British Universities', letter to Science and Public Affairs, August, 1999, p. 28.

110. 'Mathematical themes in the sculptures of John Robinson : 1 Borromean sculptures' Mathematics Today 36 (1) (2000) 6-7.

111. Review of 'History of Topology' ed. I M James, London Math Soc. Newsletter, Jan 2000, 25-27.

112. (with T. Porter) 'Mathematical Structure: Knowledge, Representation and Interpretation', in Theoria et Historia Scientiarum, the International Journal of Interdisciplinary Studies, special issue on 'Knowledge: representations and interpretations', edited W. Meyer and R. Paton. 6 (2002) 39-54.

113. 'The symbolic sculptures of John Robinson', in Theoria et Historia Scientiarum, the International Journal of Interdisciplinary Studies, issue on 'Knowledge: representation and interpretation.', edited W. Meyer and R. Paton, 6 (2002) 55-73.

114. (with İ. İçen ), 'Locally Lie subgroupoids and their Lie holonomy and monodromy groupoids', Top. and its Appl. 115 (2000) 125-138.

115. 'Mathematical themes in the sculptures of John Robinson : 2 Polyhedra' Mathematics Today 36 (6) (2000) 172-173.

116. (with M. Golasiński, T. Porter and A. P. Tonks), "Spaces of maps into classifying spaces for equivariant crossed complexes, II: The general topological group case." K-theory 23 (2001) 129-155.

117. (with İ. İçen ), 'Homotopies and automorphisms of crossed modules of groupoids', Applied Categorical Structures, 11 (2003) 185-206.

118. (with F. A. Al-Agl and R. Steiner), "Multiple categories: the equivalence of a globular and a cubical approach", Advances in Mathematics 170 (2002) 71-118.

119. (with T. Porter), "The methodology of mathematics", European Mathematical Society Newsletter June (2001) 12-14, Sept. (2001) 14-16.

120. (with K. Hardie, K. H. Kamps, T. Porter), “ The homotopy double groupoid of a Hausdorff space", Theory and Applications of Categories, 10 (2002) 71-93.

121. (with İ. İçen and O. Mucuk) 'Holonomy and monodromy groupoids', in Lie Algebroids, Banach Center Publications Institute of Mathematics, Polish Academy of Sciences, Warsaw, 54 (2001) 9-20.

122. 'Algebraic homotopy', Supplement III Encyclopaedia of Mathematics Managing Editor: M. Hazewinkel Kluwer Academic Publishers (2002) 29-31.

123. 'Exponential law in topology', Supplement III Encyclopaedia of Mathematics Managing Editor: M. Hazewinkel Kluwer Academic Publishers (2002) 142-143.

124. (with İ. İçen, and O. Mucuk), 'Examples and coherence properties of local subgroupoids', Topology and its Applications 127 (2003) 393-408.

125. (with İ. İçen) 'Towards a 2-dimensional notion of holonomy', Advances in Math. 178 (2003) 141-175.

126. (with C. D. Wensley), 'Computation and homotopical applications of induced crossed modules', J. Symbolic Computation 35 (2003) 59-72. 
127. (with M. Bullejos and T. Porter), 'Crossed complexes, free crossed resolutions and graph products of groups', Proceedings Workshop Korea 2000, Heldermann Verlag. 27 (2003) 11-26.

128. (with E. Moore, T. Porter, C. D. Wensley), 'Crossed complexes, and free crossed resolutions for amalgamated sums and HNN-extensions of groups', math.AT/0207037, Georgian Math. J. 9 (2002) 623-644.

129. 'Serie di sculture simboliche dell'universo di John Robinson', in Matematica, arte, tecnologia, cinema, edited Michele Emmer, Mirella Manaresi, SpringerVerlag Italia, Milano, 2002, 19-30.

130. 'Forum discussion', in Mathematics and Art,: Mathematical Visualisation in Art and Education, Editor Claude P. Bruter, Springer-Verlag (Berlin Heidelberg) 2002, 155-159.

131. (with G. Janelidze), 'Galois Theory and a new homotopy double groupoid of a map of spaces', Applied Categorical Structures 12 (2004) 63-80.

132. (with T. Porter), 'The intuitions of higher dimensional algebra for the study of structured space', Revue de Synthse, 124 (2003) 173-203.

133. (with P. J. Higgins), 'Cubical abelian groups with connections are equivalent to chain complexes', Homology, Homotopy and Applications, 5(1) (2003) 49-52.

134. 'Crossed complexes and homotopy groupoids as non-commutative tools for higher dimensional local-to-global problems', Proceedings of the Fields Institute Workshop on Categorical Structures for Descent and Galois Theory, Hopf Algebras and Semiabelian Categories, September 23-28, 2002, Fields Institute Communications 43 (2004) 101-130.

135. 'Les escultures simbóliques de John Robinson', Métode, Universitat de Valencia, Revista de Difusiò de la Investigaciò , Primavera 2003, no. 37, 67-71.

136. (with J. F. Glazebrook), 'Connections, local subgroupoids, and a holonomy Lie groupoid of a line bundle gerbe', Univ. Iagel. Acta Math. XLI (2003) 283-296.

137. (with H. B. Griffiths) 'Obituary: W.H. Cockcroft', Bull. London Math. Soc. 37 (2005) 149-155.

138. (with T. Porter), 'Category theory and higher dimensional algebra: potential descriptive tools in neuroscience', Proceedings of the International Conference on Theoretical Neurobiology, Delhi, February 2003, edited by Nandini Singh, National Brain Research Centre, Conference Proceedings 1 (2003) 80-92.

139. (with R. Paton and T. Porter), 'Categorical language and hierarchical models for cell systems', Computation in Cells and Tissues-Perspectives and Tools of Thought, Paton, R.; Bolouri, H.; Holcombe, M.; Parish, J.H.; Tateson, R. (Eds.) Natural Computing Series, Springer (2004) 289-303.

140. ' $k$-spaces', in Encyclopedia of General Topology, ed. K. P. Hart, J. Nagata, and J. E. Vaughan, Elsevier (2004) 189-191.

141. (with N. Ghani, A. Heyworth, C. D.Wensley), 'String rewriting systems for double coset systems', J. Symb. Comp. 41 (2006) 573-590.

142. (with K. H. Kamps and T. Porter), 'A homotopy double groupoid of a Hausdorff space II: a van Kampen theorem', Theory and Appl. Cat.14, (2005) 200-220.

143. 'John Robinson's Symbolic Sculptures: Knots and mathematics', in 'The visual mind II', edited Michele Emmer, MIT Press, (2005) 125-139.

144. 'Mathematicians struggle for truth', Letter to the Independent, Aug 17, 2005. 
145. 'Allow beautiful minds to thrive', letter in the Times HigherEducational Supplement, September 16, 2005.

146. 'Origins of Grothendieck's Pursuing Stacks', Letter, Notices American Math. Soc., 52 (8) Sept, 2005, 830.

147. (with I. C. Baianu, G. Georgescu, and J. F. Glazebrook), 'Complex nonlinear biodynamics in categories: higher dimensional algebra, and LukasiewiczMoisil Topos: transformations of neuronal, genetic and neoplastic networks', Axiomathes 16 (2006) 65-122.

148. (with T. Porter) 'Category Theory: an abstract setting for analogy and comparison', In: What is Category Theory? Advanced Studies in Mathematics and Logic, Polimetrica Publisher, Italy, (2006) 257-274.

149. 'Three themes in the work of Charles Ehresmann: Local-to-global; Groupoids; Higher dimensions', Proceedings of the 7th Conference on the Geometry and Topology of Manifolds: The Mathematical Legacy of Charles Ehresmann, Bedlewo (Poland) 8.05.2005-15.05.2005, Banach Centre Publications 76, Institute of Mathematics Polish Academy of Sciences, Warsaw, (2007) 51-63.

150. (with A. Bak, G. Minian, and T. Porter), 'Groupoid atlases: an Introduction', abstract of talk to the 'Colloque International: Charles Ehresmann: 100 ans', Amiens 7-9 October, 2005, Cah. Top. Géom. Diff. Cat. 46 (2005) 183.

151. (with A. Bak, G. Minian, and T. Porter), 'Global actions, groupoid atlases and applications', J. Homotopy and Related Structures, 1 (2006) 101-167.

152. (with T. Porter), 'Analogy, concepts and methodology in mathematics', Eureka, (September 2006) 23-27.

153. 'Groupoids, the Phragmen-Brouwer property and the Jordan curve theorem', J. Homotopy and Related Structures 1 (2006) 175-183. arXiv:math/0607229.

154. (with R. Sivera), 'Normalisation for the fundamental crossed complex of a simplicial set', J. Homotopy and Related Structures, Special Issue devoted to the memory of Saunders Mac Lane, 2 (2007) 49-79. arXiv:math/0611728.

155. 'Promoting Mathematics', MSOR Connections 7 no 2 July 2007 21-25.

156. 'John Robinson Sculptor, May 5 1935-April 6, 2007', Hyperseeing, June 2007, 3-7.

157. 'John Robinson, The Sculptor', LMS Newsletter, July 2007, p. 8.

158. (with I. C. Baianu and J. F. Glazebrook), 'Categorical Ontology of Complex Space-Time Structures: The Emergence of Life and Human Consciousness', Axiomathes, 17: (2007) 223-352.

159. (with I. C. Baianu and J. F. Glazebrook), 'A Conceptual Construction for Complexity Levels Theory in SpaceTime Categorical Ontology: Non-Abelian Algebraic Topology, Many-Valued Logics and Dynamic Systems', Axiomathes, 17 (2007).

160. (with I. C. Baianu and J. F. Glazebrook), 'A Non-Abelian Categorical Ontology and Higher Dimensional Algebra of Spacetimes and Quantum Gravity'. Axiomathes, 17 (2007) 353-408.

161. (with I. C. Baianu and J. F. Glazebrook), Categorical Ontology of Complex Systems, Meta-Systems and Levels: The Emergence of life, Human Consciousness and Society'. In: "Theory and Applications of Ontology." vol.1, R. Poli, et al., eds. 2008, Springer: Berlin. 
162. 'Remembering George Mackey', Letter, Notices American Mathematical Society, 54 no. 10 , November, 2007, p. 1280.

163. 'Mathoetic mode', Newsletter, London Mathematical Society, January 2008, p. 26.

164. 'A new higher homotopy groupoid: the fundamental globular omega-groupoid of a filtered space', Homotopy, homology and applications, 10 (2008), No. 1, pp. 327-343.

165. (with I. Morris, J. Shrimpton and C. D. Wensley), 'Graphs of Morphisms of Graphs', Electronic Journal of Combinatorics, A1 of Volume 15(1), 2008. 1-28.

166. 'Exact sequences of fibrations of crossed complexes, homotopy classification of maps, and nonabelian extensions of groups', J. Homotopy and Related Structures 3 (2008) 331-343.

167. 'Crossed complexes and homotopy groupoids as non commutative tools for higher dimensional local-to-global problems', in Michiel Hazewinkel (ed.), Handbook of Algebra, volume 6, Elsevier, 2009, pp. 83-124.

168. 'Memory evolutive systems', Axiomathes, 19 (2009) 271-280. (Review of book by A-C. Ehresmann and J. P. Vanbremeersch).

169. (with I. C. Baianu, and J. F. Glazebrook), 'Algebraic topology foundations of supersymmetry and symmetry breaking in quantum field theory and quantum gravity: a review' SIGMA (Symmetry, Integrability, and Geometry: Methods and Applications) $79 \mathrm{pp}$.

170. (with R. Sivera), 'Algebraic colimit calculations in homotopy theory using fibred and cofibred categories', Theory and Applications of Categories, 22 (2009) 222251.

171. 'Promoting mathematics', EMS Newsletter, June 2010, 17-21.

172. 'Possible connections between whiskered categories and groupoids, Leibniz algebras, automorphism structures and local-to-global questions', J. Homotopy and Related Structures, 5(1) (2010) 305-318.

173. (with R. Street), 'Covering morphisms of crossed complexes and of cubical $\omega$ groupoids are closed under tensor product', Cah. Top. Geom. Diff. Cat., 52 (2011) 188-208.

174. 'Erratum to "Possible connections between whiskered categories and groupoids, Leibniz algebras, automorphism structures and local-to-global questions ", vol. 5 (1) 2010, pp. 305-318.' J. Homotopy and Related Structures (2011) (to appear).

175. (with I. Baianu and J. F. Glazebrook), "Quantum Symmetries, Operator Algebra and Quantum Groupoid Representations: Paracrystalline Systems, Topological Order, Supersymmetry and Global Symmetry Breaking”, International Journal of Research and Reviews in Applied Sciences, 9, Issue 2 (November, 2011) 163-206.

176. (with T. Porter), "What should be the context of an adequate specialist undergraduate education in mathematics?", The De Morgan Journal 2 no. 1, (2012) 411-67.

177. (with J. F. Glazebrook), "A Career of Unyielding Exploration: In Memory of Ion C. Baianu (1947-2013)”, Quanta, (2) No 1 (2013) 1-6. 
178. (with O. Antolin Camarena), "Corrigendum to "Groupoids, the PhragmenBrouwer Property, and the Jordan Curve Theorem", J. Homotopy and Related Structures 1 (2006) 175-183." J. Homotopy and Related Structures, 4 pp.

179. "Alexander Grothendieck: some recollections", The De Morgan Forum, 28 December, 2014.

180. “Correspondence A. Grothendieck and R. Brown”, Edited M. Künzer, Documents Mathématiques (to appear).

\section{Other output}

(i) "How mathematics gets into knots", London Mathematical Society Popular Lectures Video Series, (1987) $50 \mathrm{~min}$.

(ii) "Mathematics and Knots", Exhibition of 16 A2 boards, designed by R. Brown, N. D. Gilbert, and T. Porter, Mathematics and Knots, Bangor, 1989. Exhibited at the Royal Society/Joint Mathematical Council Pop Maths Roadshow, 1989-90; British Association for the Advancement of Science, Swansea, 1990; National Science Festival, Edinburgh, 1994.

(iii) "Pivoted lines and the Möbius Band", Computer Graphics Video, Storyline by R. Brown, Programming by Ramen Sen, Mathematics and Knots / IBM UK 4 min. (1992). See the end of Pivoted lines and the Mbius Band.

(iv) "Symbolic Sculptures and Mathematics", Symbolic Sculptures and Mathematics Sculptures: John Robinson. Production: Cara Quinton. Mathematics: Ronald Brown. Edition Limitée; Centre for the Popularisation of Mathematics, 1996.

(v) Web Site Knot Exhibition Centre for the Popularisation of Mathematics, 1997, (now at http://groupoids.org.uk/popmath/cpm/exhib/knotexhib.html).

(vi) 'Raising public awareness of mathematics': This was a CDRom published for World Mathematics Year 2000 and funded by the EC. It is largely incorporated in the web site of $(\mathrm{v})$. 